\title{
Volumetric Assessment of Bone Microstructures by a 3D Local Binary Patterns -Based Method: Bone Changes with Osteoarthritis
}

\author{
Jérôme Thevenot ${ }^{1}$, Jukka Hirvasniemi ${ }^{1}$, Mikko Finnilä ${ }^{1,2}$, Petri Lehenkari ${ }^{3}$, Simo Saarakkala ${ }^{1,3}$ \\ ${ }^{1}$ University of Oulu, Oulu, Finland \\ ${ }^{2}$ University of Eastern Finland, Kuopio, Finland \\ ${ }^{3}$ Oulu University Hospital, Oulu, Finland
}

\begin{abstract}
Osteoarthritis (OA) causes progressive degeneration of articular cartilage and pathological changes in subchondral bone, conventionally assessed volumetrically using microcomputed tomography $(\mu \mathrm{CT})$ imaging in vitro. The local binary patterns (LBP) method has recently been suggested as a new alternative solution to perform analysis of local bone structures from $\mu C T$ scans. In this study, a novel 3D LBP-based method to provide a new lead in bone microstructural analysis is proposed. In addition to the detailed description of the method, this solution is tested using $\mu \mathrm{CT}$ data of OA human trabecular bone samples, harvested from patients treated with total knee arthroplasty. The method was applied to correlate the distribution of orientations of local patterns with the severity of the disease. The local orientations of the bone fibers changed along the severity of $\mathrm{OA}$, suggesting an adaptation of the bone to the disease. The structural parameters derived from the process were able to provide a new approach for the assessment of the disease, supporting the potential of this volumetric LBPbased method to assess trabecular bone changes.
\end{abstract}

Keywords- Bone structures; micro-CT; osteoarthritis; 3D analysis; LBP.

\section{INTRODUCTION}

The bone can be divided in two different structures: the trabecular bone and the cortical bone surrounding it. While the dense cortical part provides the rigidity and bending resistance of the bone, the trabeculae, corresponding to the inner part, is metabolically more active. As suggested already in the 19th century by Wolff's law [1], the trabecular bone adapts itself to environmental changes, such as daily loadings. Not only its architecture is highly correlated with strength, but it can also provide symptomatic indications of diseases [2]. Due to these characteristics, the trabecular part of the bone is at upmost interest in studies of bone adaptation to conditions such as osteoporosis (OP) or osteoarthritis (OA) [3]. While OP corresponds to a bone loss and an increase of the bone fragility [4], OA is a disease of the whole joint causing among other things - a remodeling of the subchondral bone (bone area below the cartilage) [5].

Different imaging modalities are available to assess the trabecular architecture clinically (e.g., X-ray, computed tomography (CT)). However, to perform accurate analysis of the bone structures, devices with higher resolutions are required. For this purpose, micro-CT $(\mu \mathrm{CT})$ is a good alternative, as the imaging technique is similar to the clinical CT one, but at micro-scale level instead of macro-scale level. Beside its high resolution, a main asset of $\mu \mathrm{CT}$ is its capability of provide volumetric data of microstructures [6]. While applied to bone samples, it allows to visualize the organization of the trabeculae and obtain information related to its density, and eventually on the strength of the bone. Multiple artefacts can affect the $\mu \mathrm{CT}$ scans, the main two for structure analysis being: the partial volume effect (bone not totally within the voxel) [7] and the beam hardening (inconstant brightness within the stack of images) [8]. Thus, the development of methods unaffected by these issues is a challenging topic to be addressed in bone structural analysis.

Conventional bone analysis consists on the binarization of the data from a threshold believed to represent the minimum greyscale value of the bone, to generate a $3 \mathrm{D}$ model. Then several parameters of the trabecular bone are calculated from this model to assess the bone inner architecture [6]. The main limitation of the conventional method is that the information related to the pixel values are ignored in the analysis. An alternative method using local binary patterns (LBP) [9], was recently suggested to assess trabecular bone structures [10]. The low sensitivity of the LBP for monotonic greyscale variations [9] makes it ideal for medical image processing. One of the main asset of LBP -based method on bone microarchitecture studies, is that the local distribution of patterns can be assessed not only in 2 dimensions (2D), but also in 3D by applying the analysis on multiple slices simultaneously. Recent studies applied the method in 1D and 2D on plain radiographic imaging [10-11] and 2D slice-byslice in $\mu \mathrm{CT}$ imaging [12]. The results obtained showed the potential of this new tool to assess OA, and eventually a full 3D approach was suggested [13], providing a better understanding of bone-related changes of OA.

The aim of this paper is to describe in details the 3D LBP based method previously reported [13]. The methodological concepts introduced here were developed to get a real volumetric investigation of the bone by multi-slice analysis. The method was applied to human OA bone sample scans, to correlate different structural parameters derived from the method, with the severity of OA from histopathology grading. 


\section{Bone STRUCTURAL ANALYSIS USING LBP METHOD}

\section{A. Basics of 3D LBP applied to bone structural analysis}

The neighbors of a central (studied) voxel are evaluated to assess if some have an equal or higher grey level values than the studied voxel. Based on the location of these occurrences (markers), a local pattern is derived from a corresponding weight matrix. The pattern depicts the local structure surrounding the studied voxel and it can provide an information on the nature of the neighborhood: such as edges, contours and flat regions. The local structure $s$ of each neighbor within a region of interest is calculated by the following function:

$$
\mathrm{LBP}=\sum_{k=0}^{n-1} s\left(g_{k}-g_{c}\right) 2^{k}, \quad s(x)= \begin{cases}1, & x \geq 0 \\ 0, & x<0,\end{cases}
$$

where $\mathrm{n}$ is the amount of neighbors evaluated, $g_{k}$ the grey level value of the k-th neighbor, and $g_{c}$ the value of the central (studied) voxel. The final amount of different possible patterns is $2^{\text {n }}$, being $\approx 67$ million in the example in Fig. 1 ( $n=26$ neighbors). However, it has to be noted that some patterns are redundant in structural analysis, and that they need to be grouped, based on their identical local structures.

Typically, in image texture analysis using LBP method, every pixels are assessed and a $2^{\mathrm{n}}$-sized histogram is generated, with $\mathrm{n}$ being the amount of neighbors considered in the analysis. For 3D LBP applied to structural analysis of volumetric data, the concept is similar except that the method is applied solely to the relevant voxels within or at the edge of the bone, ignoring the "empty spaces" in the analysis.

\section{B. Spherical approach and grouping of patterns}

To improve the cubic representation of the method (Fig. 1) by taking into consideration the distance between voxels, a sphere is created with equidistant neighbors fitted on it. While the studied voxel is the center of the sphere, the grey level value of each neighbor is interpolated from its surrounding voxels values.
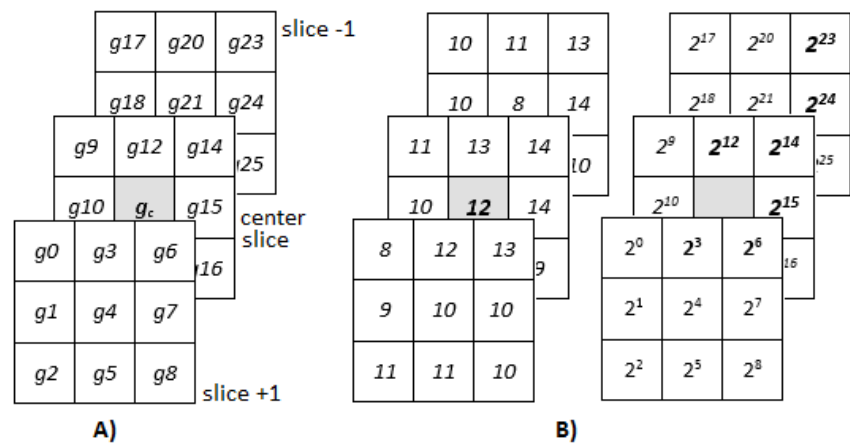

Fig. 1 Cubic 3D-LBP. A) A center pixel $g_{c}$ and its 26 neighbors; B) basic LBP: original picture in left with grey center pixel, weight matrix in right with relevant values bolded $\left(I d=2^{3}+2^{6}+2^{12}+2^{14}+2^{15}+2^{23}+2^{24}=25,219,144\right)$.
The number of voxels from which the neighbors are interpolated depends on the location and varies between 1 (neighbor at the center of a voxel) and 8 voxels (4 voxels in 2 consecutive slices). The amount of neighbors required to cover the volumetric aspect of the analysis is based on the radius of the sphere. A minimum of 26 neighbors can be suggested for a sphere with radius 1 , this number requires to be higher for bigger spheres.

Due to the huge amount of possible patterns ( $>67$ millions) in volumetric analysis, a grouping of redundant / similar patterns is required. Therefore, we propose to group the patterns by their main orientations using Principal Component Analysis (PCA). This method can provide both an angle corresponding to the elevation between $0^{\circ}$ and $90^{\circ}$, and an angle corresponding to the azimuth between $0^{\circ}$ and $180^{\circ}$ (Fig.2A). Eventually, the elevation represents the angle between the horizontal and vertical planes, while the azimuth angle provides information in the horizontal plane. To perform PCA, the position of the markers on the sphere is used and the main orientation of the pattern is then derived from it (Fig.2B). The axis obtained from PCA is then converted in elevation and azimuth angles.
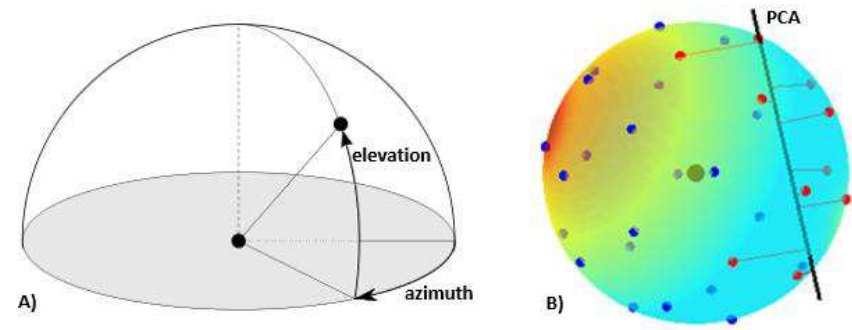

Fig. 2 PCA to evaluate elevation and azimuth angles. A) Representation of the angles; B) PCA analysis from the markers (red dots).

Before grouping the patterns from the angles obtained, the consistency of the PCA is assessed. This is performed by evaluating how sparse are the markers towards the axis obtained by PCA. For this purpose, if any non-marker (blue dots in the Fig. 2B) is closer to the axis than the markers, it suggests that the pattern is non-uniform. A representation of patterns uniformity is provided in Fig. 3.
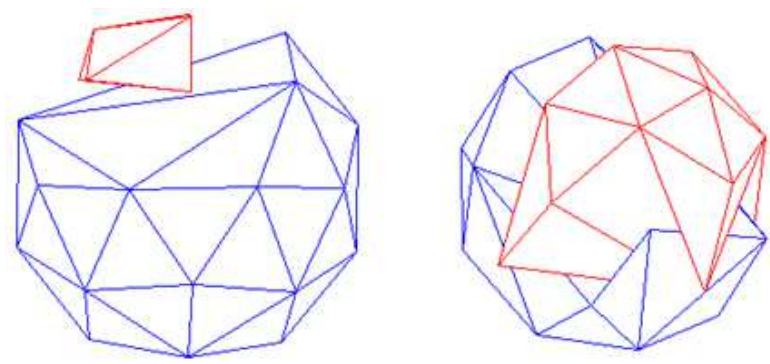

Fig. 3 Patterns uniformity. Left: Uniform pattern; right: non-uniform pattern (the blue and red volumes cross each other's). 


\section{EXPERIMENT: APPLICATION TO HUMAN OA SAMPLES}

\section{A. Sample preparation, OA grading and imaging}

The samples and their preparation have been described in details previously [12-13]. Briefly, 24 osteochondral samples, obtained from tibial plateus, were prepared from 14 patients with OA (age $76 \pm 9$ years), treated with total knee arthroplasty at Oulu University Hospital. Sample collection and their use were approved by the Ethical Committee of the Northern Ostrobothnia Hospital District, Oulu, Finland.

The severity of OA was analyzed from the histological sections of the samples using the standardized OARSI grading system [14]. The final OARSI grade was calculated by averaging the values obtained by three evaluators. Samples were scanned with a $\mu \mathrm{CT}$ device at isotropic voxel size of 27.8 $\mu \mathrm{m}$ (Skyscan 1172, Bruker microCT, Kontich, Belgium). The scanned trabecular bone was located below the subchondral plate of the proximal tibia.

The $\mu$ CT scans were then segmented with a fully automatic custom-made algorithm developed in a numerical computing environment and programming language (MATLAB version R2013b, MathWorks, Natick, Massachusetts, USA). The script separated the bone and its edges from the irrelevant empty spaces using a threshold value derived from the average minimum value returned by Otsu method [15] applied to the whole stack of data. An example of a segmented slice using the method is provided in Fig. 4.

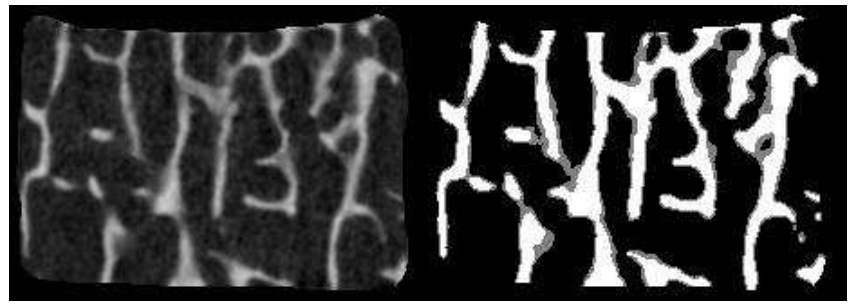

Fig. 4 Data segmentation. Left: original slice; right: segmented data, in white the bone and in grey its edges assessed from multi-slices analysis.

\section{B. Patterns grouping}

After the segmentation, the 3D LBP -based analysis was performed on the relevant voxels to assess the patterns structures, their 3D orientation and the amount of markers of each of them. The method was applied using a sphere with radius 1 and 26 neighbors. Since only the proximal-distal axis was known, only the elevation angle was assessed in the analysis. The elevation angles were grouped every $15^{\circ}$, from $0^{\circ}$ (horizontal axis) to $90^{\circ}$ (vertical, parallel to the distalproximal axis). The uniformity test was applied for all the patterns to keep only the relevant data in the structural analysis. A distribution of the local elevation angles is provided in Fig. 5.

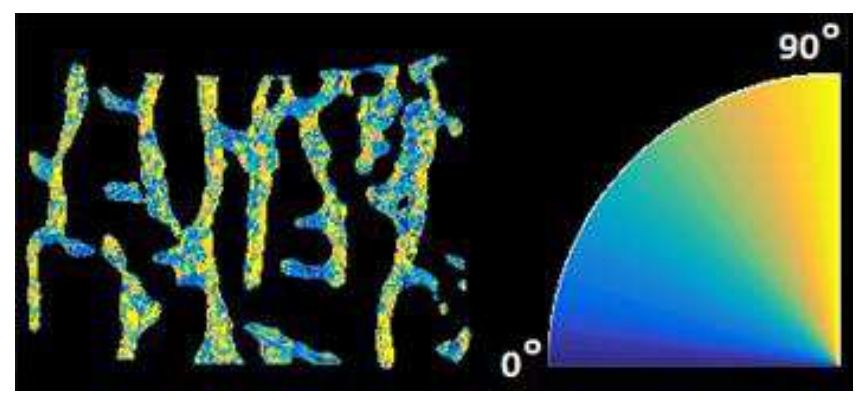

Fig. 5 Distribution of elevation angles within a $\mu \mathrm{CT}$ slice.

\section{Definition of the structural parameters}

All the data processing part was performed with a custommade algorithm developed in MATLAB. For each sample, various parameters were assessed from the 3D LBP method:

- The mean amount of markers: corresponds to the mean amount of markers (1-26) for all the local patterns of the sample pooled together.

- The amount of different patterns: for 26 neighbors, the maximum amount of different patterns is $\approx 67$ million. This parameter provides a count of all the different local patterns recognized within the sample.

- The entropy of local patterns: describes the randomness of local patterns and is calculated as follows:

$$
E=-\sum_{i} P_{i} \log _{2} P_{i}
$$

where $P_{i}$ contains the count of a specific local pattern $i$ occurring in the data. If an image contains only one local pattern, the entropy of the patterns within the image is zero.

- The entropy of elevation: describes the randomness of elevation values. Similar equation than (2), where Pi contains the count of a specific elevation angle i occurring in the data. If an image contains only one elevation angle, the entropy of the elevation angle within the image is zero.

- The homogeneity of the elevation: Describe spatial distribution of the elevation angle and provides indication on its continuity. An angle-level co-occurrence matrix (ALCM) was first calculated with volumetric rotational invariance, to assess the spatial distribution of the elevation angle. The homogeneity of the elevation was then calculated from the ALCM as such:

$$
H_{\text {elevation }}=\sum_{i, j} \frac{\operatorname{ALCM}(i, j)}{1+|i-j|}
$$

where each element $(i, j)$ in the ALCM is the sum of the occurrences that a voxel with elevation $i$ has a voxel with elevation $j$ in its vicinity (one voxel-size radius). Eventually, a high value of homogeneity of the elevation corresponds to data with consecutive voxels with similar elevation angles.

- The average elevation: provides the mean elevation angle of the sample when the orientations of all the studied voxels are pooled together. 


\section{Structural parameters versus severity of the disease}

The OARSI grades obtained were considered as the ground truth for OA severity with a distribution from 0.89 to 6.25 (mean $3.51 \pm 1.69$ ). The statistical analyses were performed using SPSS statistical software (version 20.0; SPSS, Chicago, Illinois, USA). For all analysis, Pearson's linear correlation coefficients were calculated between the OARSI grade and the parameters derived from 3D LBP-based method (see Table I).

Table 1 Pearson correlation coefficients between the structural parameters and the OARSI grades. $\mathrm{N}=24$

\begin{tabular}{llll}
\hline & \multicolumn{1}{c}{ Edges } & Bone & Total \\
\hline Mean amount markers & $0,79 * * *$ & $0,83 * * *$ & $0,82 * * *$ \\
Amount different patterns & $0,43 *$ & $0,68 * * *$ & $0,68 * * *$ \\
Entropy patterns & $0,67 * * *$ & $0,79 * * *$ & $0,79 * * *$ \\
Entropy elevation & $0,49 *$ & $0,57 * *$ & $0,53 * *$ \\
Homogeneity elevation & $-0,58 * *$ & $-0,75 * * *$ & $-0,71 * * *$ \\
Average elevation & $-0,37$ & $-0,37$ & $-0,36$
\end{tabular}

$* \mathrm{p}<0.05, * * \mathrm{p}<0.01, * * * \mathrm{p}<0.001$

Similarly to the results obtained previously in the study of Hirvasniemi et al. [11] and from the previous 2D [12] and 3D [13] analysis of the same $\mu \mathrm{CT}$ data, the entropy of local patterns is proportionate to the increase of OA severity. These results suggests that "new" local patterns occur with an increase of OA level, which could be explained by the appearance of bone sclerosis at higher OARSI grades [14]. This hypothesis is further supported by the increase of amount of different patterns with OA. With OA, the trabecular bone tends to compensate the degeneration of the overlying cartilage by changing the orientation of its fibers, indicating a local regulation of bone metabolism (i.e., osteoclast regulation). As suggested in the Table I, the average elevation of the voxels decreases with OA, meaning that some fibers parallel to the cartilage orientation are created, making connections between vertical fibers. These "bridges" are clearly visible in the Fig. 5 (in blue), as they connect the "original" fibers (in yellow). Due to its characteristic, the homogeneity of the elevation provides better information than its entropy, as it assess the continuity of the angles while the entropy assess the general distribution.

The slight difference in the reported results here compared to the previous study [13] is related to the segmentation which was performed fully automatically in this study, from an algorithm based on structure continuity to extract edges in 3D. The analysis of edges is highly influenced by the partial volume effect and the restricted amount of considered voxels can explain the slightly "weaker" results. However, this distinction between edges and bone should be further investigated with larger data and also for other bone conditions such as OP.

\section{Conclusion}

This study describe a novel 3D LBP - based method to perform fully automatic volumetric bone structural analysis of $\mu \mathrm{CT}$ data. While conventional bone structural analysis ignores the grey level value information of the data, the method here is directly based on the local distribution of the voxel values. Furthermore, the results obtained from the LBPderived structural parameters were able to assess the local changes in the trabecular bone with $\mathrm{OA}$, and as such, validate the potential of this approach for microstructural bone analysis. Further developments of the method should be performed to adapt it to clinical modalities and to provide a better estimation of the disease severity.

\section{ACKNOWLEDGMENT}

This study was supported by Infotech Oulu, the Academy of Finland, the European Research Council (ERC grant no. 336267) and the strategic funding of the University of Oulu.

\section{CONFLICT OF INTEREST}

The authors declare that they have no conflict of interest.

\section{REFERENCES}

1. Wolff J (1892) The law of bone remodelling, Berlin, 1986: SpringerVerlag, 1892

2. Roesler H (1987) The history of some fundamental concepts in bone biomechanics, J. Biomech 20:1025-1034

3. Zhang ZM, Li ZC, Jiang LS, Jiang SD, Dai LY (2010) Micro-CT and mechanical evaluation of subchondral trabecular bone structure between postmenopausal women with osteoarthritis and osteoporosis, Osteoporos Int 21:1383-1390

4. Riggs BL (1991) Overview of osteoporosis, West J. Med. 154:63-77

5. Hunter DJ, Felson DT (2006) Osteoarthritis, BMJ 332:639-642

6. Bouxsein ML, Boyd SK, Christiansen BA et al (2010) Guidelines for assessment of bone microstructure in rodents using micro-computed tomography, J. Bone Miner. Res. 25:1468-1486

7. Souza A, Udupa JK, Saha PK (2005) Volume rendering in the presence of partial volume effects, IEEE Trans. Med. Imaging 24:223-235

8. Nakashima Y, Nakano T (2014) Optimizing contrast agents with respect to reducing beam hardening in nonmedical X-ray computed tomography experiments, J. Xray Sci. Technol. 22:91-103

9. Pietikäinen M, Hadid A, Zhao G, Ahonen T (2011) Computer Vision Using Local Binary Patterns, Springer, 2011.

10. Houam L, Hafiane A et al (2014) One dimensional local binary pattern for bone texture characterization, Pattern Anal. Appl.17:179-193

11. Hirvasniemi J, Thevenot J, Immonen V et al (2014) Quantification of differences in bone texture from plain radiographs in knees with and without osteoarthritis, Osteoarthr Cartilage 22:1724-1731

12. Thevenot J et al (2015) Local Binary Patterns to Evaluate Trabecular Bone Structure from Micro-CT Data: Application to Studies of Human Osteoarthritis, ECCV 2014 Workshops Part II, LNCS 8926:63-79

13. Finnilä M, Thevenot J, Aho OM et al (2016) Association between subchondral bone structure and osteoarthritis histopathological grade, J Orthop Res. DOI 10.1002 [Epub ahead of print]

14. Pritzker KP, Gay S, Jimenez SA et al (2006) Osteoarthritis cartilage histopathology: grading and staging, Osteoarthr Cartilage 14:13-29

15. N. Otsu (1979) A treshold selection method from gray-level histograms, IEEE Trans. Syst., Man, Cybern., Syst. 9:62-66 